\title{
Comprensión y Uso Docente de Gráficos Estadísticos por Futuros Profesores para Promover Competencias para la Sostenibilidad
}

\author{
Claudia Vásquez \\ cavasque@uc.cl \\ https://orcid.org/0000-0002-5056-5208 \\ Pontificia Universidad Católica de Chile (PUC) \\ Villarrica, Chile.
}

Recibido: 16/09/2020 Aceptado: 18/02/2021

\begin{abstract}
Resumen
En este artículo se analizan las preguntas que plantean 48 futuros profesores de Educación Primaria chilenos para promover en sus estudiantes la comprensión de gráficos y el desarrollo de competencias para la sostenibilidad. El estudio se realizó a partir del análisis de gráficos estadísticos presentes en los medios de comunicación, en el contexto de la COVID-19. Nos basamos en investigaciones que describen los conocimientos necesarios para que el profesorado pueda movilizar en sus estudiantes un conjunto de competencias para desenvolverse como ciudadanos críticos en el análisis de datos. Con este propósito, se proporcionó a los participantes un conjunto de tareas apoyadas en gráficos estadísticos de la prensa frente a los cuales debieron diseñar preguntas a proponer a sus futuros estudiantes, con el fin de abordar competencias fundamentales de la comprensión de gráficos, así como algunas competencias clave para educar en sostenibilidad. Las preguntas propuestas por los futuros profesores se han categorizado en función del nivel de lectura que permiten alcanzar. Los resultados muestran la necesidad de fortalecer el conocimiento asociado a la comprensión de gráficos estadísticos, y sobre el uso docente de éstos como herramienta para contribuir al desarrollo de competencias que permitan formar ciudadanos educados en sostenibilidad.
\end{abstract}

Palabras clave: Futuros profesores. Educación Primaria. Gráficos estadísticos. Educación para el desarrollo sostenible. Lectura de gráficos.

\section{Compreender e ensinar o uso de gráficos estatísticos por futuros professores para promover habilidades de sustentabilidade}

\section{Resumo}

Este artigo discute as questões colocadas por 48 futuros professores de escolas primárias chilenas para promover nos seus alunos a compreensão dos gráficos e o desenvolvimento de competências para a sustentabilidade. O estudo foi realizado com base na análise de gráficos estatísticos presentes nos meios de comunicação, no contexto da COVID-19. Baseamo-nos na investigação que descreve os conhecimentos necessários para os professores mobilizarem nos seus alunos um conjunto de competências a desempenhar como cidadãos críticos na análise de dados. Para este efeito, os participantes receberam um conjunto de tarefas apoiadas por gráficos estatísticos da imprensa contra os quais tiveram de conceber perguntas a propor aos seus futuros alunos, a fim de abordarem competências fundamentais na compreensão dos gráficos, bem como algumas competências-chave para a educação em sustentabilidade. As questões propostas pelos futuros professores foram categorizadas de acordo com o nível de 
leitura que permitem alcançar. Os resultados mostram a necessidade de reforçar os conhecimentos associados à compreensão dos gráficos estatísticos, e sobre a utilização destes gráficos como ferramenta para contribuir para o desenvolvimento de competências que permitam formar cidadãos educados em sustentabilidade.

Palavras chave: Futuros professores. Educação primária. Gráficos estatísticos. Educação para o desenvolvimento sustentável. Leitura de gráficos.

\title{
Prospective teachers' understanding and teaching use of statistical graphs to promote sustainability skills
}

\begin{abstract}
We analyse the questions posed by 48 Chilean prospective primary school teachers to promote the understanding of graphs and the development of sustainability skills in their students. The study was carried out using the analysis of statistical graphs present in the media, in the context of COVID-19. We base on research that describes the knowledge necessary for teachers to mobilize in their students a set of skills to perform as critical citizens in data analysis. With this purpose, the participants were provided with a set of tasks supported by statistical graphs from the press for which they had to formulate questions to propose to their students, in order to promote fundamental competences in understanding graphs, as well as some key competences for educating in sustainability. The questions proposed by the prospective teachers have been categorized according to the reading level they allow to achieve. The results show the need to strengthen the knowledge associated with the understanding of statistical graphs, and on the use of these graphs as a tool to contribute to the development of competences oriented to educate citizens in sustainability.

Key words: Future teachers. Primary education. Statistical graphics. Education for sustainable development. Reading graphics.
\end{abstract}

\section{Introducción}

Nos encontramos en medio de una situación de extrema gravedad derivada de la pandemia ocasionada por el nuevo virus SARS-CoV-2, que nos afecta en diversos aspectos sociales, sanitarios, medioambientales, económicos, que están relacionados. Esto ha provocado que en los últimos meses estemos bombardeados con datos y distintos tipos de representaciones gráficas a través de los medios de prensa, televisión y redes sociales que, como ciudadanos, debemos saber interpretar, con el fin de tomar decisiones que de una u otra manera impactan en nuestra vida.

Hoy cobra relevancia lo señalado por H. G. Wells a comienzos del siglo XX: “el comprender promedios, máximos y mínimos algún día será tan necesario para una ciudadanía eficiente como lo es hoy la habilidad de leer y escribir" (Wells, 1911/1903, p. 204). Estas ideas incluso nos hacen cuestionar si hemos actuado con lentitud frente a esta necesidad, pues 
hoy nos vemos enfrentados a la urgente necesidad de contar con una Educación Estocástica (Batanero, 2019) que impulse un pensamiento crítico. Dicha educación debe a su vez generar cambios de comportamiento en las personas para que tomen decisiones de manera informada y actúen a favor de fomentar una cultura de respeto en una sociedad que cada día se vuelve más compleja. Un ejemplo son las normas sanitarias para el autocuidado, que contribuya de alguna manera a mitigar los efectos ocasionados por esta pandemia.

Por consiguiente, es importante contar con datos reales y herramientas estocásticas que permitan realizar interpretaciones y análisis adecuados para la toma de decisiones, así como para identificar la información no relevante o que no se ha comunicado adecuadamente. Todavía más, si consideramos que recientemente la Organización para la Cooperación y el Desarrollo Económico (OCDE) ha reconocido la necesidad de avanzar en el desarrollo de habilidades y conocimientos que permitan formar ciudadanos alfabetizados en el análisis de datos, es decir, capaces de "extraer información significativa de los datos, comprender qué significan los datos, incluyendo cómo leerlos de manera apropiada, extraer conclusiones, así como reconocer cuándo se utilizan de manera engañosa o inapropiada" (OCDE, 2019, p. 5).

De acuerdo con Batanero, Díaz, Contreras y Roa (2013), hoy más que nunca es imperativo desarrollar el sentido estadístico en el aula escolar, entendido como la unión de la cultura estadística y el razonamiento estadístico. Dicho sentido implica la asimilación de ideas estadísticas fundamentales como la comprensión de gráficos, así como el desarrollo del razonamiento estadístico para la toma de decisiones, con el fin de interpretar, evaluar críticamente y, cuando sea pertinente, expresar opiniones respecto a la información estadística. De esta manera, todos los ciudadanos podrán entender, por ejemplo, qué esta pasando y qué podemos hacer para mitigar los efectos de la pandemia de la COVID-19.

Si bien son muchas las herramientas matemáticas y técnicas estadísticas que se están empleando para trabajar y comprender la gran avalancha de datos que emergen de este fenómeno, resulta interesante analizar un aspecto clave en la comprensión de esta pandemia: la comunicación de estos datos.

Al revisar diversos medios de comunicación podemos observar que la información se está comunicando principalmente a través de gráficos estadísticos que buscan presentar de manera rápida los datos, primordialmente a través de gráficos de líneas, de barras, de sectores y pictogramas. Por tanto, en este escenario es importante considerar la transnumeración 
(Pfannkuch \& Wild, 2004), componente del razonamiento estadístico, que consiste en descubrir características de un conjunto de datos a partir de su representación gráfica. Este proceso implica una transformación de los datos recolectados en diversas representaciones con el fin de promover la comprensión de una situación real. Por consiguiente, los gráficos estadísticos desempeñan un rol fundamental para la comprensión de la pandemia, pues, por un lado, permiten organizar y presentar/comunicar visualmente los datos; y por otro, como herramienta para el análisis/exploración de los datos, sus relaciones, patrones de variabilidad y tendencia de los mismos. Así pues, es necesario contar con ciudadanos cultos, capaces de leer críticamente, comprender e interpretar los gráficos estadísticos elementales a los que a diario nos vemos expuestos (Sharma, 2013).

Pero, ¿cómo llevar estos gráficos estadísticos al aula escolar?, ¿cómo aprovecharlos para desarrollar la competencia gráfica en los estudiantes, y a la vez generar una comprensión y toma de conciencia del fenómeno COVID-19? Esto constituye un gran desafío para el profesorado, sobre todo si consideramos que la enseñanza de los gráficos estadísticos se encuentra más centrada en aquellos aspectos asociados a la construcción de gráficos que en la interpretación y comprensión de la información que éstos buscan comunicar (González, Espinel \& Ainley, 2011).

En Chile, la enseñanza de los gráficos estadísticos se aborda de manera gradual a lo largo de toda la Educación Primaria (MINEDUC, 2012) y en la Educación Secundaria (MINEDUC, 2015), con el propósito de que:

Los educandos desarrollen los conocimientos, habilidades y actitudes que les permitan: pensar en forma reflexiva, evaluando y utilizando información y conocimientos, de manera sistemática y metódica, para la formulación de proyectos y resolución de problemas; comprender y utilizar conceptos y procedimientos matemáticos básicos en la resolución de problemas cotidianos, y apreciar el aporte de la matemática para entender y actuar en el mundo (MINEDUC, 2009, p. $10)$.

De este modo, el currículo escolar chileno busca contribuir a desarrollar una cultura estadística en los estudiantes (los ciudadanos del mañana), entendida desde la perspectiva de Gal y Murray (2011) como la competencia para interpretar y evaluar críticamente la información estadística, así como la capacidad de formular y comunicar a otros una opinión razonada sobre dicha información. 
En consideración a lo expuesto, el propósito del presente estudio es indagar la comprensión y el uso docente de futuros profesores de Educación Primaria de los gráficos estadísticos vinculados a la COVID-19, presentes en medios de comunicación, cuando buscan promover en sus estudiantes no solo la comprensión de los gráficos estadísticos sino también competencias que les permitan reflexionar sobre cuestiones vinculadas a problemáticas reales a nivel local y global. Con este objetivo en mente, se describen y clasifican las respuestas de 48 futuros profesores de Educación Primaria a dos tareas de evaluación. Estas competencias para la sostenibilidad (pensamiento sistémico, anticipación, normativa, estratégica, colaboración, pensamiento crítico, autoconciencia y resolución de problemas) deben ser desarrolladas por todos los alumnos (a distintos niveles según la edad), y no reemplazan a las competencias de las diferentes áreas como por ejemplo matemática, pero las comprenden y tienen un mayor alcance (Rychen, 2003).

Los resultados de este estudio servirán para la posterior organización y diseño de actividades de formación, a través de las cuales se replantee el enfoque tradicional de enseñanza de la estadística en el aula escolar -en el que, por lo general, se observa un predominio de lo algorítmico- por otro enfoque centrado en el desarrollo de competencias que promuevan una educación para la sostenibilidad. Ello se logrará a través de un pensamiento crítico que permita "empoderar y equipar a las generaciones presentes y futuras para satisfacer sus necesidades mediante un enfoque equilibrado e integrado de las dimensiones económica, social y ambiental del desarrollo sostenible" (Leicht, Heiss \& Byun, 2018, p.7). No hay que olvidar que "la estadística es inseparable de sus aplicaciones y su justificación final es su utilidad en la resolución de problemas externos a la propia estadística" (Batanero \& Díaz, 2011, p. 21). Por consiguiente, la actual necesidad de desarrollar competencias para el desarrollo sostenible se constituye en un propósito para enseñar estadística y a su vez la estadística se convierte en un medio para formar en sostenibilidad.

\section{Comprensión de gráficos estadísticos}

Los gráficos estadísticos surgen como una herramienta para presentar datos visualmente, con el propósito de facilitar su comprensión y su análisis (González et al., 2011). De ahí que todo ciudadano del siglo XXI debiera ser capaz de leer críticamente los gráficos estadísticos provenientes de diversos medios de comunicación. De acuerdo con Arteaga, 
Batanero, Cañadas y Contreras (2011), ello implica no solo una lectura literal, sino también la capacidad para identificar tendencias, variabilidad, asociaciones de los datos y detectar posibles errores que pueden llevar a distorsionar la información representada. Desde esta perspectiva estos autores plantean que para poder leer e interpretar un gráfico son necesarios, aunque no suficientes, los siguientes elementos estructurales:

- El título y las etiquetas: indican el contenido contextual y cuáles son las variables en él representadas.

- El marco del gráfico: incluye los ejes, escalas, y marcas de referencia en cada eje. Dicho marco proporciona información sobre las unidades de medida de las magnitudes representadas. Puede haber diferentes tipos de marcos y sistemas de coordenadas (lineales, cartesianas bidimensionales o multidimensionales, polares).

- En los gráficos hay que tener también en cuenta sus especificadores, es decir, los elementos visuales usados para representar los datos, como los rectángulos (en el histograma) o los puntos (en el diagrama de dispersión).

Es importante fomentar la comprensión de gráficos estadísticos, entendida como la capacidad de leer e interpretar la información presente en ellos, ya que es un elemento clave para desarrollar una cultura estadística en el aula (Arteaga, Batanero, Contreras \& Cañadas, 2016). Esta comprensión debe permitir a los estudiantes, a partir del trabajo con datos provenientes de contextos cercanos y significativos, tomar conciencia sobre su propia acción en el entorno y tomar decisiones que modifiquen su estilo de vida hacia uno sostenible (Vásquez \& García-Alonso, en prensa). No obstante, la investigación respecto a los gráficos estadísticos y su enseñanza pone de manifiesto que ésta a menudo se centra en el proceso de construcción, dando poca atención a su interpretación (González et al., 2011). En consecuencia, es de importancia formar al profesorado, de manera que sea capaz de:

Planificar clases destinadas a desarrollar habilidad para la construcción de tablas y lectura e interpretación de gráficos en los distintos niveles, con el propósito de desarrollar el pensamiento crítico de sus estudiantes frente a afirmaciones hechas a partir de un conjunto de datos y, además, su capacidad de detectar el uso inadecuado de la información (MINEDUC, 2012, p. 111).

La lectura y comprensión de gráficos es un campo bastante explorado y que cada vez cobra mayor presencia en la agenda de investigación en Educación Estadística, ofreciendo 
desde hace varias décadas diversos análisis respecto de lo que implica su comprensión (Aoyama, 2007; Aoyama \& Stephen, 2003; Bertin, 1967; Curcio, 1987; Friel, Curcio \& Brigth, 2001; Gerber, Boulton-Lewis \& Bruce, 1995; Shaughnessy, Garfield \& Greer, 1996). En lo que sigue se hace una síntesis del resumen que sobre esta investigación realizan Arteaga et al. (2011).

Para Bertin (1967) los gráficos son objetos semióticos complejos, cuya lectura se debe dar de manera gradual, iniciándose por una identificación externa del tema al que refiere, que abarca la comprensión del título, las etiquetas y de qué trata el gráfico; luego debe dirigirse hacia una identificación interna de las variables que se encuentran representadas, el significado del tales variables y sus escalas; y finalmente, debe realizarse una percepción de correspondencia que permita obtener conclusiones sobre los niveles de cada variable y sus relaciones con la realidad representada. En definitiva, se trata de que quien está leyendo el gráfico comprenda los datos que se exponen a partir de la correspondencia entre los aspectos externos e internos previamente identificados. A partir de los supuestos anteriores, el autor define los siguientes tres niveles implicados en la lectura de un gráfico:

- Extracción de los datos: este nivel es el más elemental y considera solo leer exactamente lo que hay en el gráfico, sin realizar operaciones ni comparaciones de datos.

- Extracción de las tendencias: este nivel considera la percepción entre la relación de subconjuntos de datos que intervienen en el gráfico, que pueden ser definidos a priori o visualmente, e implica operar con los datos o bien compararlos.

- Análisis de la estructura de los datos: este nivel incluye la comparación de tendencias o agrupamientos y el efectuar predicciones.

Gerber et al. (1995) plantean una visión más compleja de la comprensión de gráficos, y consideran siete niveles, en función de las competencias de los estudiantes para interpretarlos, que van desde aquellos estudiantes que no se centran en los datos, sino en su conocimiento del mundo en forma imprecisa, hasta estudiantes que realizan extrapolaciones y predicciones a partir de los datos expuestos en el gráfico.

A partir de estos estudios, algunos investigadores han propuesto y ampliado los niveles de lectura, pues conciben la comprensión gráfica como un proceso gradual que se desarrolla a 
partir del uso de una variedad de gráficos presentes en contextos problemáticos diversos, y que implica:

La habilidad de los lectores de derivar significado desde los gráficos creados por otros o por ellos mismos y los diferentes niveles de preguntas provocan diferentes niveles de comprensión. Además, varios factores críticos influyen en la comprensión de las gráficas: el propósito de usar gráficas, las características de la tarea, las características de la disciplina y las características del lector (Friel et al., 2001, p. 132).

A partir de estas ideas, se consideran cuatro niveles de lectura de gráficos tomados de los trabajos de Curcio (1987), Friel et al. (2001) y Shaughnessy et al. (1996), que corresponden a: “leer los datos" (lectura literal del gráfico sin interpretar la información contenida en el mismo); "leer dentro de los datos" (interpretación e integración de los datos en el gráfico); "leer más allá de los datos" (predicciones e inferencias a partir de los datos sobre informaciones que no se reflejan directamente en el gráfico); y "leer detrás de los datos" (valorar críticamente el método de recogida de datos, su validez y fiabilidad, así como las posibilidades de extensión de las conclusiones).

Ahora bien, cuando lo que se desea es realizar una valoración crítica de los gráficos, el nivel de "leer detrás de los datos" se analiza por Aoyama \& Stephen (2003) y Aoyama (2007), subdividiéndose en tres:

- Nivel racional/literal: los estudiantes pueden leer valores particulares en gráficos y deducir las tendencias; pueden explicar significados contextuales literalmente usando las características del gráfico, pero no pueden sugerir ninguna interpretación alternativa.

- Nivel crítico: los estudiantes pueden leer gráficos, comprender el contexto y evaluar la confiabilidad de la información, cuestionándola a veces, pero no pueden sugerir hipótesis alternativas.

- Hipótesis y nivel de modelado: los estudiantes pueden leer gráficos, aceptar y evaluar la información, y pueden sugerir sus propias hipótesis o modelos explicativos.

Esta jerarquización de niveles de comprensión de gráficos, de acuerdo con González et al. (2011), puede servir de marco para formular preguntas en un contexto pedagógico, orientado no solo a la comprensión de gráficos sino también a reflexionar sobre cuestiones 
vinculadas a problemáticas reales y al desarrollo de un pensamiento crítico para la toma de

decisiones. Al mismo tiempo, los distintos niveles de comprensión de gráficos deben contribuir a proporcionar las herramientas para que los estudiantes puedan discriminar entre información relevante y no relevante, o aquella que no se ha comunicado adecuadamente (Shaughnessy et al., 1996). De este modo, todas las personas tendrían no solo la oportunidad de desarrollar el sentido estadístico en el aula escolar, sino también adquirir conocimientos, competencias, valores y actitudes con los que puedan contribuir a un desarrollo sostenible a través de una educación de calidad que fomente la reflexión y la crítica (Vásquez, 2020).

\section{Hacia una educación estadística para educar en sostenibilidad}

En las últimas décadas se observa un creciente interés a nivel mundial por incorporar en el currículo escolar el estudio de la estadística y la probabilidad desde temprana edad (Batanero, Burril \& Reading, 2011), con el propósito de que todos los ciudadanos adquieran las habilidades y conocimientos necesarios para desenvolverse en el mundo actual como ciudadanos críticos, de modo que puedan participar de manera informada y conformar sociedades cada vez más democráticas (e.g. Batanero, 2019; Engel, 2019; Vásquez, 2020; Vásquez \& Rojas, 2020; Wild, Utts \& Horton, 2018).

En este sentido, diversos autores y organismos recomiendan enfocar la enseñanza de la estadística a partir de contextos reales, adecuados a la edad y a la etapa escolar de los alumnos, que tengan significado para ellos, de manera tal que les permita avanzar hacia el aprendizaje de conceptos estocásticos, el empleo de técnicas de cálculo, y a la vez mejorar sus capacidades de argumentación, formulación de conjeturas y reflexión en torno a dichos contextos. Esto al mismo tiempo de desarrollar el sentido de los datos, pues "lo que importa no son los datos, sino las respuestas y los conocimientos que buscamos en los datos" (Gal, 2019, p. 4).

Sin embargo, no es una tarea fácil de llevar a cabo, pues, "el contexto no está presente automáticamente en el aula -tenemos que introducirlo" (Gal, 2019, p. 3). Pero ¿cómo incluir el contexto para enseñar estadística en el aula escolar?, ¿qué contextos utilizar? Las respuestas a este último interrogante pueden ser muy variadas. No obstante, un contexto acerca del cual resulta de vital importancia poder reflexionar es el desarrollo sostenible, pues desde hace ya algunos años el mundo (nuestra casa común) se encuentra en medio de una verdadera crisis, por lo que es necesario actuar y desarrollar competencias que permitan contar con ciudadanos 
alfabetizados en esta temática. La Educación Estadística se constituye como una herramienta poderosa que contribuye directamente al desarrollo de las competencias clave de sostenibilidad las cuales son transversales e impactan directamente en los Objetivos de Desarrollo Sostenible (ODS) (Figura 1) y, por ende, va en ayuda directa a formar ciudadanos educados, que contribuyan a crear un mejor presente y transitar hacia un futuro mejor.

Figura 1. Relación entre competencias clave de sostenibilidad y los ODS.



Fuente: Tomado de Vásquez, 2020, p. 7.

Este objetivo, implica reorientar la enseñanza de la estadística en el aula escolar hacia una enseñanza contextualizada que transite hacia una educación estadística para educar en sostenibilidad (Vásquez, 2020). Desde este enfoque, es necesario que todas las personas tengan la oportunidad de desarrollar el sentido estadístico (Batanero et al., 2013), además de adquirir conocimientos, competencias, valores y actitudes con los que puedan contribuir a un desarrollo sostenible. Más aún si consideramos que en el último informe del Estudio Regional Comparativo y Explicativo para América Latina y el Caribe (UNESCO, 2020) se evidencia que en el área de Matemáticas los conceptos asociados a la Educación para el Desarrollo Sostenible (EDS) aparecen a un nivel declarativo y no son utilizados por los profesores en el 
diseño de sus clases, por lo que "persiste el desafío de llevar estos elementos a aspectos más concretos, y de vincularlos en forma integral a la programación de contenidos y objetivos disciplinares" (UNESCO, 2020, p. 80).

A tal efecto, la educación estadística tiene mucho por aportar focalizando su enseñanza a partir del abordaje de problemáticas provenientes de contextos reales y locales vinculados con los ODS, con el propósito de desarrollar competencias que empoderen al profesorado y, por ende, a sus estudiantes para reflexionar sobre cuestiones vinculadas al desarrollo sostenible. Lo anterior permitirá, por un lado, que los estudiantes conozcan y tomen conciencia de la EDS y los ODS, y reflexionen respecto de lo que pueden hacer para contribuir a alcanzarlos desde las propias acciones (tomar decisiones); y por otro lado, otorgar sentido al aprendizaje tanto de la estadística como de la probabilidad, permitiendo así una mayor interiorización de lo aprendido (Vásquez, 2020; Vásquez \& García-Alonso, en prensa).

\section{Investigaciones sobre comprensión gráfica en futuros profesores de Educación Primaria}

Gran parte de las investigaciones que analizan la comprensión gráfica de los futuros profesores de Educación Primaria se centran en la lectura y construcción de gráficos. Por ejemplo, Burgess (2002) analizó el conocimiento estadístico de 30 futuros profesores de este nivel educativo, de los cuales solo seis fueron capaces de producir gráficos adecuados a partir de la información entregada y la mitad logró interpretar el gráfico y relacionarlo con el contexto implicado.

Bruno y Espinel (2005) estudian los errores cometidos por 29 futuros profesores de Educación Primaria cuando deben construir diagramas de barras e histogramas a partir de una lista de datos proporcionada. Dentro de los errores más frecuentes se encuentran intervalos mal representados, omisión de intervalos de frecuencia nula, uso de rectángulos no adosados en variables continuas, confusión de la frecuencia con el valor de la variable entre otros. Posteriormente, Espinel (2007) analiza la interpretación de gráficos de futuros profesores y estudiantes universitarios. Los resultados muestran una mayor dificultad para la interpretación por parte de los futuros profesores.

Monteiro y Ainley (2006) analizan el nivel de lectura de gráficos estadísticos tomados de la prensa en 218 futuros profesores de primaria de Brasil e Inglaterra. Los resultados evidencian dificultades para llevar a cabo una correcta lectura de gráficos. No obstante, 
muestran que la lectura de gráficos se ve influenciada por los conocimientos estadísticos y otros elementos relacionados como la experiencia personal sobre el contexto del cual provienen los datos. Asimismo, se observa la presencia de capacidad para pensar críticamente sobre aspectos de los datos presentados en los gráficos y para justificar sus ideas basándose en estadísticas u otras fuentes de información.

Arteaga (2011) evalúa la lectura e interpretación de gráficos estadísticos y su uso para obtener conclusiones en 207 futuros profesores de primaria. Los resultados muestran que parte de los participantes presentan errores y dificultades para la correcta construcción de los gráficos, invalidando su uso para el problema propuesto. De igual manera, se evidencia un bajo nivel en la lectura de gráficos, pues un porcentaje importante de los futuros profesores no leen los gráficos realizados, ni son capaces de interpretarlos en relación a la pregunta planteada. Siguiendo la anterior investigación, Batanero, Arteaga y Ruíz (2010) analizan los gráficos producidos por 93 futuros profesores de primaria cuando se enfrentan a un proyecto abierto de análisis de datos en el que deben comparar dos variables estadísticas. Los resultados evidencian que solo algunos de los participantes logran producir un gráfico adecuado para alcanzar conclusiones, mientras que otros no consiguen un nivel de lectura suficiente o bien no logran una conclusión completa en relación con la pregunta planteada. Posteriormente, Arteaga et al. (2016) analizan la construcción de gráficos estadísticos en 207 futuros profesores de primaria al resolver una tarea en la que debían comparar tres pares de distribuciones. Se identificaron en las construcciones conflictos semióticos relacionados con los convenios de construcción, selección, sentido numérico y errores conceptuales.

García-Alonso y Bruno (2019) analizan los niveles de lectura de gráficos en 90 futuros profesores de primaria y 110 estudiantes de secundaria. Se evidencia lo compleja que puede resultar esta tarea para ambos grupos de participantes sobre todo cuando la información gráfica se presenta distribuida en dos gráficos. Rodríguez y Sandoval (2012) evalúan el nivel de lectura de gráficos de 47 profesores en ejercicio y 44 futuros profesores de Educación Primaria. Se evidencia un pobre nivel de lectura tanto de los profesores en ejercicio como de los futuros profesores.

Sin duda estos estudios proporcionan información relevante para el presente trabajo que busca complementar los resultados encontrados hasta el momento añadiendo la dimensión 
de la Educación para el Desarrollo Sostenible como contexto real y cercano al alumnado. A continuación, se describen la metodología seguida de los resultados.

\section{Metodología}

Se utiliza una metodología de tipo cualitativa y descriptiva (Bisquerra, 2009), basada en el análisis de contenido (Krippendorff, 2013), ya que se busca describir y analizar los tipos de preguntas que plantean los futuros profesores de Educación Primaria para promover la comprensión de gráficos y el desarrollo de competencias de sostenibilidad en sus estudiantes.

Participantes y contexto. El estudio se desarrolló con 48 futuros profesores de Educación Primaria de una universidad chilena, los cuales se encontraban cursando por primera vez la asignatura de estadística básica y su didáctica durante el primer semestre académico de 2020. Se trata de una materia didáctico-disciplinar, que busca entregar una sólida formación a los futuros profesores respecto de los contenidos de estadística y probabilidad que deberán enseñar.

Recolección de datos. Se proporcionó a los participantes un conjunto de tareas (Cuadros 1 y 2) apoyadas en gráficos estadísticos de la prensa y se les puso en una situación ficticia a través del uso de viñetas (Schoenberg \& Ravdal, 2000), solicitándoles diseñar preguntas a proponer a sus futuros estudiantes. Estas preguntas tienen el fin de abordar competencias fundamentales de la comprensión de gráficos (lectura e interpretación, construcción y evaluación crítica de gráficos), así como algunas competencias clave de sostenibilidad (anticipación, normativa, pensamiento crítico y autoconciencia).

\section{Cuadro 1.}

Tarea 1 propuesta a los futuros profesores

\section{Tarea 1 - Ventiladores totales vs disponibles}

Constanza es la profesora de matemática de un $6^{\circ}$ año de primaria. Ella consciente de la importancia de la enseñanza de la estadística en contexto, quiere abordar con sus estudiantes el siguiente objetivo de aprendizaje: "leer e interpretar gráficos y comunicar sus conclusiones".

Además, Constanza quiere que sus estudiantes reflexionen acerca de las consecuencias de sus acciones, sobre los valores, percepciones y acciones propias, sobre el rol que cada uno tiene en la comunidad local y en la sociedad en el contexto de la crisis sanitaria de la COVID-19. Con estos propósitos en mente Constanza se ha planteado mostrar, analizar e interpretar el gráfico de la Figura siguiente con sus estudiantes. 
Constanza te pide ayuda, y te solicita lo siguiente:

a) Tu opinión acerca de si el gráfico seleccionado te parece apropiado para abordar el objetivo de aprendizaje señalado. Justifica tu respuesta.

b) Que propongas 4 preguntas -que atiendan a los aspectos antes señalados- para que sus estudiantes respondan en relación con el gráfico.

Número de ventiladores mecánicos totales versus disponibles en Chile hasta finales de mayo de 2020 (Fuente: MINSAL - Chile).

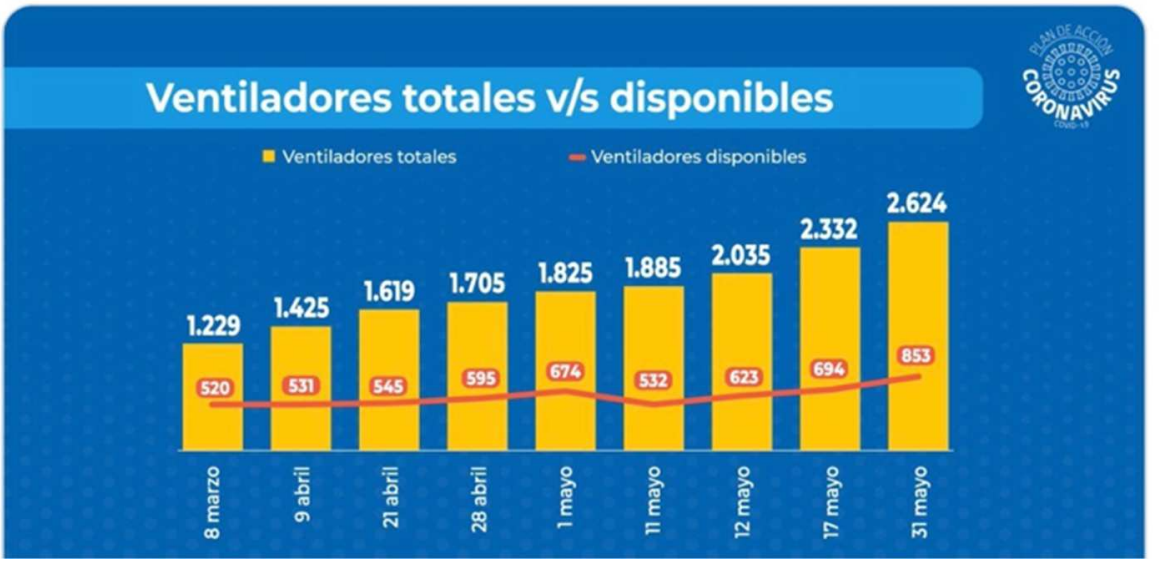

Cuadro 2.

Tarea 2 propuesta a los futuros profesores

\section{Tarea 2 - Duración del coronavirus en superficies}

Francisco es el profesor de matemática de un $3^{\circ}$ año de primaria y quiere abordar con sus niños y niñas el siguiente objetivo de aprendizaje: "construir, leer e interpretar pictogramas y gráficos de barra simple con escala, en base a información recolectada o dada"

Francisco encontró en internet los siguientes datos representados en la figura que muestran la duración del COVID-19 (medida en horas y días) en distintas superficies y que le interesa analizar con sus estudiantes.

A partir de dicha información Francisco decide construir un pictograma y luego un gráfico de barras de manera tal que sus estudiantes puedan alcanzar el objetivo de aprendizaje propuesto. Pero aún no sabe bien qué tipo de preguntas puede plantear para que sus estudiantes reflexionen acerca del autocuidado en el contexto de la crisis sanitaria de la COVID-19.

¿Qué tipo de preguntas podría plantear Francisco a partir de la información presente en tales gráficos? Señala cuatro posibles preguntas.

Adaptación de la duración de la COVID-19 en distintas superficies (Fuente: www.elmundo.es). 


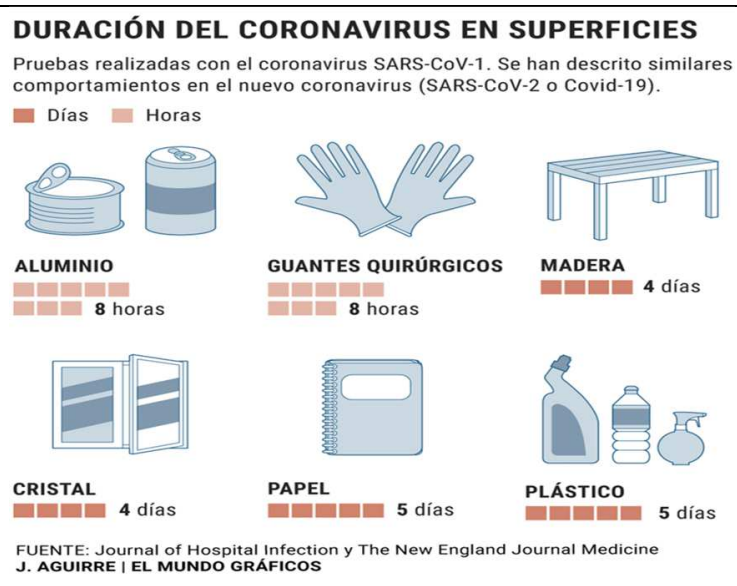

Ambas tareas forman parte de un taller formativo sobre "comprensión de gráficos estadísticos en el contexto de la COVID-19" que fue respondido por los participantes del estudio por escrito y de forma individual. Para ello, contaron con un tiempo de 90 minutos. Cabe señalar que previo a la realización del taller formativo los futuros profesores contaron con una sesión de 90 minutos de sensibilización respecto de la importancia del estudio de la estadística en contexto y su vínculo con la EDS así como la necesidad de incorporar un contexto para la enseñanza de la estadística en el aula escolar.

Procedimiento de análisis. Una vez recogidos los datos, se analizaron las respuestas y argumentos dados por los futuros profesores, utilizando como método el análisis de contenido (Krippendorff, 2013), e identificando distintos grados de cuestionamiento y análisis de acuerdo con los cuatro niveles de comprensión descritos por Curcio (1987), Shaughnessy et al. (1996) y Friel et al. (2001). Para ello, se consideraron los siguientes pasos:

- Lectura individual de cada una de las respuestas para familiarizarse con el contenido y poder organizar y estructurar la información en cada tarea.

- En el caso de la Tarea 1, se analizaron en primer lugar las respuestas dadas a la pregunta 1 en la que interesa conocer la opinión de estos futuros profesores acerca de si el gráfico seleccionado les parece o no apropiado para abordar el objetivo de aprendizaje señalado. Los argumentos vinculados a tales respuestas se analizaron a la luz de los elementos estructurales propuestos por Arteaga et al. (2011), pues son necesarios tenerlos en consideración al momento de realizar una adecuada lectura del gráfico. Luego se procedió a analizar y clasificar las preguntas propuestas por los participantes. 
- En el caso de la Tarea 2, se analizaron y clasificaron las preguntas propuestas.

- Finalmente, se seleccionaron ejemplos específicos para las distintas preguntas que componen las tareas analizadas.

\section{Resultados}

Como se ha indicado, el objetivo de este estudio fue analizar la comprensión y el uso docente que hacen futuros profesores de Educación Primaria de los gráficos estadísticos, cuando quieren promover competencias para la sostenibilidad en sus estudiantes. Para exponer los resultados, se ha decidido hacerlo de acuerdo al orden de cada una de las tareas propuestas.

\section{Resultados de la Tarea 1 - Ventiladores totales vs disponibles}

El gráfico de barras y de líneas presentado en el Cuadro 1 informa de dos variables, el número de ventiladores totales en Chile y el número de ventiladores disponibles entre el 8 de marzo y el 31 de mayo.

Al preguntar a los futuros profesores sobre su opinión acerca de si el gráfico seleccionado les parece apropiado para abordar el objetivo de aprendizaje señalado, 40 de los 48 futuros profesores $(83,3 \%)$ consideran que el gráfico no es apropiado para abordar el objetivo de "leer e interpretar gráficos y comunicar sus conclusiones". Dentro de los argumentos para apoyar esta opción, se encuentran los recogidos en la Tabla 1, que se han agrupado en tres categorías. Tales argumentos se vinculan prácticamente en su totalidad con elementos estructurales (Arteaga et al., 2011), principalmente con aquellos referidos al marco del gráfico, es decir a la falta de etiquetas en las variables y omisión de la escala. Respecto a lo primero, hacemos notar que se han utilizado colores para identificar las variables y se indica el significado de los colores en la parte superior del gráfico.

\section{Tabla 1.}

Opiniones de los futuros profesores respecto al gráfico presentado en el Cuadro 1.

\section{Argumentos}

Frecuencia (\%)

$\begin{aligned} & \text { No es apropiado porque falta información, ya que no se indica el nombre } \\ & \text { de la variable analizada en los ejes. }\end{aligned}$
$\begin{aligned} & \text { En el gráfico faltan las etiquetas de los ejes x e y; además, se omite la } \\ & \text { escala utilizada. }\end{aligned}$




\begin{tabular}{lc}
\hline $\begin{array}{l}\text { Considero que este es un tema que no se debe abordar con estudiantes de } \\
\text { esta edad. }\end{array}$ & $2(4,1 \%)$ \\
No responde & $8(16,7 \%)$ \\
\hline Total & $\mathbf{4 8 ( 1 0 0 \% )}$ \\
\hline
\end{tabular}

Fuente: elaborado por el autor

En lo que respecta a las 192 preguntas planteadas por los 48 futuros profesores para sus alumnos, éstas se han agrupado de acuerdo con los niveles de lectura de los gráficos estadísticos recogidos en Curcio (1987), Shaughnessy et al. (1996) y Friel et al. (2001), como se resumen en la Tabla 2.

\section{Tabla 2.}

Distribución de las preguntas planteadas para la Tarea 1 de acuerdo con los niveles de lectura.

\begin{tabular}{lc}
\hline Niveles de lectura & Frecuencia (\%) \\
\hline Nivel 1. Leer los datos & $80(41,7 \%)$ \\
Nivel 2. Leer dentro de los datos & $78(40,6 \%)$ \\
Nivel 3. Leer más allá de los datos & $34(17,7 \%)$ \\
Nivel 4. Leer detrás de los datos & $0(0 \%)$
\end{tabular}

Total $192(100 \%)$

Fuente: elaborado por el autor

La lectura e interpretación de gráficos es fundamental para una adecuada comprensión de los mismos. En este sentido, los distintos niveles de lectura permiten explorar distintos niveles de cuestionamiento. A partir de la Tabla 2, se observa que 158 de las 192 preguntas planteadas se encuentran distribuidas entre el nivel 1 y el nivel 2 con una diferencia porcentual muy pequeña.

Un $41,7 \%$ de las preguntas planteadas se encuentran en el nivel 1 de "leer los datos", es decir, son preguntas en las que el lector debe simplemente extraer datos desde el gráfico, ya sea por medio de la lectura del título, variables, etiquetas y valores. Ejemplos de este tipo de preguntas son la siguientes, en que se solicita el nombre de la variable representada, el valor que corresponde a una frecuencia o una descripción general del gráfico:

¿Qué representan las barras y las líneas en el gráfico? (FP 17) ¿Qué día hubo 674 ventiladores disponibles? (FP 3) ¿Qué información entrega el gráfico? (FP 48) 
En lo que refiere al nivel 2 de "leer dentro de los datos" un 40,6\% de las preguntas planteadas se encuentra en este nivel, es decir, el estudiante para responder debe interpretar, integrar, interpolar o encontrar relaciones entre los datos del gráfico, ya sea a través de la realización de comparaciones o mediante operaciones matemáticas entre los valores representados en el gráfico. Algunos ejemplos de este tipo de preguntas son las siguientes, en que se pide realizar cálculos con los datos del gráfico:

¿Cuál es la diferencia entre los ventiladores disponibles el 31 de mayo y el 8 de marzo? (FP 2)

Para el día 1 de mayo hay 1705 ventiladores totales y 595 ventiladores disponibles. Teniendo en cuenta estos datos, ¿cuántos ventiladores han sido utilizados el día 1 de mayo? (FP 5)

El día 8 de marzo había 1129 ventiladores totales y para el día 9 de abril había 1425 ventiladores totales. ¿En cuánto aumentó el número de ventiladores totales? (FP 6)

En el nivel 3 de "leer más allá de los datos" se encuentran un 17,7\% de las preguntas. En ellas se requiere que el estudiante realice predicciones, extrapolaciones, inferencias o hipótesis a partir de los datos que se presentan en el gráfico. Ejemplos de este tipo de pregunta son las que se exponen a continuación, donde se pide extrapolar uno o más valores de datos no representados:

¿Cómo crees que será la gráfica después del 31 de mayo? (FP 1)

De acuerdo con lo que se observa en el gráfico, ¿qué se podría esperar que ocurra con la diferencia entre los ventiladores totales y los disponibles después del 31 de mayo? (FP 11)

¿Qué cantidad de ventiladores disponibles estimas que había antes del 8 de marzo? (FP 20)

Por último, cabe destacar que ninguno de los futuros profesores planteó preguntas que se pudieran clasificar en el nivel 4 de "leer detrás de los datos", que son precisamente aquellas preguntas que contribuyen a desarrollar un pensamiento crítico, a comprender el contexto en el cual se encuentra la información, de manera que los estudiantes puedan formular sus propias hipótesis, y por qué no, tomar conciencia del contexto y sus implicaciones. Es precisamente este nivel uno de los que puede aportar al desarrollo de competencias de sostenibilidad. 


\section{Resultados de la Tarea 2 - Duración del coronavirus en superficies}

Al preguntar a los futuros profesores sobre qué tipo de preguntas propondrían con la información entregada en el Cuadro 2, sus preferencias distribuyen, de acuerdo con los niveles de Curcio (1987), Shaughnessy et al. (1996) y Friel et al. (2001), como muestra la Tabla 3. Cabe precisar que un 93,8\% de los futuros profesores, antes de plantear las preguntas señaló que, para poder construir el gráfico, Francisco debería dar el tiempo de duración (variable del gráfico) en una misma unidad de medida (horas) para poder establecer comparaciones entre los distintos tipos de superficie.

Tabla 3.

Distribución de las preguntas planteadas para la Tarea 2 de acuerdo con los niveles de lectura, Niveles de lectura Frecuencia (\%)

Nivel 1. Leer los datos $93(48,4 \%)$

Nivel 2. Leer dentro de los datos $97(50,5 \%)$

Nivel 3. Leer más allá de los datos $0(0 \%)$

Nivel 4. Leer detrás de los datos $2(1 \%)$

Total

Al igual que en la Tarea 1, donde se observaba bastante similitud en la cantidad de preguntas para los niveles 1 y 2 (Tabla 2), en la Tarea 2 también se aprecia este equilibrio entre ambos niveles, con un 48,4\% y 50,5\% respectivamente (Tabla 3 ). Sin embargo, en la Tarea 2 no se encuentran preguntas que puedan ser clasificadas como nivel 3, pero sí hay un par de preguntas (1\%) que pertenecen al nivel 4.

En lo que respecta al nivel 1 de "leer los datos" un 48,4\% de las preguntas planteadas se encuentran en este nivel de lectura, ya que se pueden responder fácilmente mirando la información a representar en el gráfico. Algunos ejemplos de este tipo de preguntas son las siguientes, similares a las planteadas en la primera tarea:

¿Qué información entrega el gráfico? (FP 48)

¿Cuál es el tiempo de duración del coronavirus en la madera? (FP 31) ¿En qué tipos de superficies se ha estudiado el tiempo de duración del coronavirus? (FP 5) 
En el segundo nivel de "leer dentro de los datos" se han clasificado un 50,5\% de las preguntas planteadas por los futuros profesores, pues son preguntas en que el estudiante debe realizar ciertas comparaciones para dar respuesta. Algunos de estos tipos de preguntas se presentan a continuación:

¿En qué tipo de superficie el coronavirus dura más tiempo? (FP 2)

¿Dónde permanece menos tiempo el coronavirus: aluminio, guantes, madera, cristal, papel o plástico? (FP 25)

¿Cuántas horas más permanece el coronavirus en una botella de plástico que en una de cristal? (FP 6)

No se encontraron preguntas que cumplieran las características propias del nivel 3 de “leer más allá de los datos”, es decir, preguntas en que se deban realizar predicciones o hipótesis a partir de los datos. Estas preguntas son más sencillas de plantear en la Tarea 1, donde el gráfico corresponde a una serie de tiempo.

A diferencia de la Tarea 1, en la que se observa ausencia de preguntas que puedan ser clasificadas en el nivel 4 de "leer detrás de los datos", para la Tarea 2 se observan dos preguntas que permiten realizar una interpretación crítica del contenido del gráfico, como son:

¿A qué crees que se debe que el coronavirus perdure más en unas superficies que en otras? ¿Cómo podemos prevenir el contagio si a diario estamos en contacto con este tipo de superficies? (FP 6)

¿Por qué consideras que hay diferencias en los tiempos de duración del coronavirus en distintas superficies? ¿qué cuidados o precauciones debemos tomar de acuerdo con los distintos tipos de superficies? (FP 42)

Si bien estas preguntas son bastante similares, dejan entre ver un nivel de cuestionamiento mayor y orientado a una toma de consciencia y de comprensión del contexto. Para responderlas, el alumno al que van dirigidas debería buscar información contextual sobre el tema a diferencia de las otras preguntas que estaban directamente relacionadas con la información presente en los gráficos.

\section{Consideraciones finales e implicaciones para la formación del profesorado}

En este estudio se indagó en los tipos de preguntas que plantean futuros profesores de educación primaria para promover la comprensión de gráficos y el desarrollo de competencias para la sostenibilidad en sus estudiantes. Para ello, se utilizaron gráficos presentes en medios de prensa vinculados con la actual crisis sanitaria de la COVID-19. Los resultados muestran 
que un gran porcentaje de las preguntas planteadas por los futuros profesores son características del nivel 1 ("leer los datos") y nivel 2 ("leer dentro de los datos") descritos por Curcio (1987), Shaughnessy et al. (1996) y Friel et al. (2001). Esto puede deberse a que las preguntas van dirigidas a niños de primaria y los futuros profesores han considerado que deben ser preguntas sencillas de responder. Pero también podrían implicar, al igual que en otros estudios con futuros profesores de primaria (Burgess, 2002; Monteiro \& Ainley, 2006; Arteaga, 2011; García-Alonso \& Bruno, 2019), un nivel de lectura de gráficos insuficiente en los participantes que no son capaces de plantear preguntas más avanzadas. Estos resultados son preocupantes, pues de los 48 futuros profesores participantes en el estudio tan solo 2 de ellos plantearon preguntas propias el nivel 4 de ("leer detrás de los datos").

Por otro lado, al preguntarles acerca de si el gráfico planteado en la primera tarea era o no adecuado para el objetivo de aprendizaje señalado, los futuros profesores se centran únicamente en la carencia de algunos elementos estructurales descritos en Arteaga et al. (2011) para argumentar sus respuestas, olvidando la importancia de la interpretación de los gráficos (González et al., 2011) y la necesidad de vincular la información presente en el gráfico con preguntas que den realce al contexto de donde provienen los datos, para generar un pensamiento crítico que contribuya a conformar una sociedad mejor formando ciudadanos educados para la sostenibilidad. No debemos olvidar que

La estadística requiere de una forma diferente de pensar, porque los datos no son sólo números, ellos son números en un contexto. En matemáticas el contexto oscurece la estructura. En análisis de datos, el contexto proporciona significado (Moore \& Cobb, 1997, p. 801).

Los resultados de esta investigación evidencian la necesidad de avanzar en el desarrollo de competencias que permitan a los futuros profesores concebir la educación estadística como una herramienta poderosa para la interpretación de datos, la toma de decisiones, donde la conexión con otras disciplinas y con temáticas diversas se dé de manera natural. En definitiva, se trata de avanzar hacia una educación estadística que permita desarrollar el sentido estadístico en el aula escolar.

Por tanto, es urgente contar con procesos de formación que desarrollen en el profesorado los conocimientos y competencias para promover la comprensión de los gráficos estadísticos en sus estudiantes y sean capaces de plantear preguntas acordes con los diferentes 
niveles de lectura de gráficos propuestos por Curcio (1987), Shaughnessy et al. (1996) y Friel et al. (2001).

En conclusión, la triada "educación estadística-formación del profesorado-educación para el desarrollo sostenible" debe ser considerada como parte fundamental para formar ciudadanos educados para la sostenibilidad en el contexto escolar. Esta triada dotará a los profesores de las competencias profesionales necesarias para desarrollar la EDS con capacidad transformadora en las aulas de modo que repercuta en toda la sociedad. Esta necesidad se muestra en las palabras emitidas, recientemente, por el Secretario General de la Organización de Naciones Unidas:

Este año en que el mundo despliega sus datos para hacer frente a un desafío común, aprovechemos el día mundial de la estadística para poner de relieve el papel de las estadísticas en la promoción del desarrollo sostenible para todos (Guterres, 2020).

Esto sin duda constituye un gran desafío para las instituciones formadoras de profesores y el profesorado en activo, pues ellos son un agente de cambio poderoso, que impacta en los distintos niveles educativos (educación infantil, educación primaria y educación secundaria). Se requiere pues, de manera urgente reorientar la enseñanza de la estadística hacia una enseñanza en contexto que permita a los ciudadanos de hoy y mañana comprender adecuadamente la información estadística para afrontar los desafíos actuales y futuros de un mundo complejo y cambiante.

\section{Agradecimientos}

Trabajo realizado en el marco del proyecto FONDECYT No 1200356 financiado por la Agencia Nacional de Investigación y Desarrollo del Gobierno de Chile.

\section{Referencias}

Aoyama. K. (2007). Investigating a hierarchy of students' interpretations of graphs. International Electronic Journal of Mathematics Education, 2(3), 298-318.

Aoyama, K. \& Stephens, M. (2003). Graph interpretation aspects of statistical literacy: A Japanese perspective. Mathematics Education Research Journal, 15(3), 3-22. https://doi.org/10.1007/BF03217380

Arteaga, P. (2011). Evaluación de conocimientos sobre gráficos estadísticos y conocimientos didácticos de futuros profesores. Tesis Doctoral. Universidad de Granada. 
Arteaga, P., Batanero, C., Cañadas, G. \& Contreras, J. M. (2011). Las tablas y gráficos estadísticos como objetos culturales. Números, 76, 55-67.

Arteaga, P., Batanero, C., Contreras, J. M. \& Cañadas, G. (2016). Evaluación de errores en la construcción de gráficos estadísticos elementales por futuros profesores. Revista Latinoamericana de Investigación en Matemática Educativa, 19(1), 15-40. https://doi.org/10.12802/relime.13.1911

Batanero, C. (2019). Treinta años de investigación en educación estocástica : Reflexiones y desafíos. En J. M. Contreras, M. M. Gea, M. M. López-Martín, \& E. Molina-Portillo (Eds.), Actas del Tercer Congreso Internacional Virtual de Educación Estadística. Universidad de Granada. www.ugr.es/local/fqm126/civeest.html.

Batanero, C., Arteaga, P. \& Ruíz, B. (2010). Análisis de la complejidad semiótica de los gráficos producidos por futuros profesores de educación primaria en una tarea de comparación de dos variables estadísticas. Enseñanza de las Ciencias, 28(1), 141-154.

Batanero, C., Burrill, G. \& Reading, C. (Eds.) (2011). Teaching statistics in school mathematics. Challenges for teaching and teacher education. A joint ICMI and IASE study. New York: Springer. https://doi.org/10.1007/978-94-007-1131-0

Batanero, C. \& Díaz, C. (2011). Estadística con proyectos. Granada: Departamento de Didáctica de la Matemática.

Batanero, C., Díaz, C., Contreras, J. M. \& Roa, R. (2013). El sentido estadístico y su desarrollo. Números. 83, 7-18.

Bertin, J. (1967). Semiologie graphique. París: Gauthier-Villars.

Bisquerra, R. (2009). Metodología de la investigación educativa (2 ${ }^{\text {a }}$ edición). Madrid: La Muralla.

Bruno, A. \& Espinel, M. C. (2005). Recta numérica, escalas y gráficas estadísticas: un estudio con estudiantes para profesores. Formación del Profesorado e Investigación en Educación Matemáticas, 7, 57-85.

Burgess, T. (2002). Investigating the "data sense" of preservice teachers. En B. Phillips (Ed.), Proceedings of the Sixth International Conference on Teaching Statistics (pp. 1-6). Cape Town: IASE.

Curcio, F. R. (1987). Comprehension of mathematical relationships expressed in graphs. Journal for Research in Mathematics Education, 18(5), 382-393. https://doi.org/10.5951/jresematheduc.18.5.0382

Engel, J. (2019). Cultura estadística y sociedad. En J. M. Contreras, M. M. Gea, M. M. LópezMartín y E. Molina-Portillo (Eds.), Actas del Tercer Congreso Internacional Virtual de Educación Estadística (pp. 1-18). Granada: Grupo FQM-126. www.ugr.es/local/fam126/civeest.html.

Espinel, C. (2007). Construcción y razonamiento de gráficos estadísticos en la formación de profesores. Investigación en Educación Matemática 11, 99-119.

Friel, S., Curcio, F. \& Bright, G. (2001). Making sense of graphs: critical factors influencing comprehension and instructional implications. Journal for Research in Mathematics Education, 32(2), 124-158. https://doi.org/10.2307/749671 
Gal, I. (2019). Understanding statistical literacy: About knowledge of contexts and models. En J. M. Contreras, M. M. Gea, M. M. López-Martín y E. Molina-Portillo (Eds.), Actas del Tercer Congreso International Virtual de Educación Estadística. www.ugr.es/local/fqm126/civeest.html.

Gal, I. \& Murray, S. T. (2011). Responding to diversity in users' statistical literacy and information needs: Institutional and educational implications. Statistical Journal of the International Association for Official Statistics, 27(3-4), 185-195. https://doi.org/ 10.3233/SJI-2011-0730.

García-Alonso, I. \& Bruno, A. (2019). Lectura de gráficos estadísticos y tareas numéricas en alumnado de secundaria y futuros profesores. En J. M. Marbán, M. Arce, A. Maroto, J. M. Muñoz-Escolano y Á. Alsina (Eds.), Investigación en Educación Matemática XXIII (pp. 313-322). Valladolid: SEIEM.

Gerber, R., Boulton-Lewis, G. \& Bruce, C. (1995). Children s understanding of graphic representation of quantitative data. Learning and Instruction 5, 70-100. https://doi.org/10.1016/0959-4752(95)00001-J

González, M. T., Espinel, M. C. \& Ainley, J. (2011). Teachers' Graphical Competence. In C. Batanero, G. Burrill, \& C. Reading (Eds.), Teaching Statistics in School Mathematics Challenges for Teaching and Teacher Education A Joint ICMI IASE Study (pp. 187-197). New York: Springer. https://doi.org/10.1007/978-94-007-1131-0_20

Guterres, A. (2020). Conectar el mundo con datos en los que podemos confiar. Día Mundial de la estadística. https://www.un.org/es/observances/statistics-day.

Krippendorff, K. (2013). Content analysis: an introduction to its methodology. London: SAGE.

Leicht, A., Heiss, J. \& Byun, W.J. (2018). Issues and trends in education for sustainable development. Paris: UNESCO Publishing

MINEDUC (2009). Ley General de Educación. Santiago de Chile: Unidad de Curriculum y Evaluación.

MINEDUC (2012). Bases curriculares 2012: Educación básica matemática. Santiago de Chile: Unidad de Curriculum y Evaluación.

MINEDUC (2015). Bases curriculares para la educación media. Unidad de Curriculum y Evaluación: Santiago de Chile.

Monteiro, C. \& Ainley, J. (2006). Student teachers interpreting media graphs. En A. Rossman, $\&$ B. Chance (Eds.), Proceedings of the Seventh International Conference on Teaching Statistics (pp. 1-6). Salvador de Bahía: ISI/IASE.

Moore, D. \& Cobb, G. (1997). Mathematics, statistics, and teaching, American Mathematical Monthly, 104, 801-823. https://doi.org/10.1080/00029890.1997.11990723

OCDE (2019). OECD Future of Education and Skills 2030: OECD Learning Compass 2030. Paris: OCDE. $\quad$ http://www.oecd.org/education/2030-project/teaching-andlearning/learning/core-foundations/Core Foundations_for_2030 concept_note.pdf 
Pfannkuch, M. \& Wild, C. (2004). Towards an understanding of statistical thinking. In J. B. Garfield \& D. Ben-Zvi (Eds.), The challenge of developing statistical literacy, reasoning and thinking (pp. 17-46). Springer Netherlands. https://doi.org/10.1007/1-4020-2278-6_2

Rodríguez, F. \& Sandoval, P. (2012). Habilidades de codificación y descodificación de tablas y gráficos estadísticos: un estudio comparativo en profesores y alumnos de pedagogía en Enseñanza Básica. Avaliação, 17(1), 207-235. https://doi.org/10.1590/S1414$\underline{40772012000100011}$

Rychen, D.S. (2003). Competencias clave: Abordar desafíos importantes en vida. Rychen, D.S. y Salganik, L.H. (Eds). Competencias clave para una vida exitosa y una sociedad sana. (pp. 63-107). Cambridge, MA, Hogrefe y Huber.

Schoenberg, N. \& Ravdal, H. (2000). Using vignettes in awareness and attitudinal research. International Journal of Social Research Methodology, 3(1), 63-74. https://doi.org/10.1080/136455700294932.

Sharma, S. (2013). Assessing students ' understanding of tables and graphs : Implications for teaching and research. International Journal of Educational Research and Technology, 4(4), 51-70.

Shaughnessy, J. M., Garfield, J. \& Greer, B. (1996). Data handling. En A. J. Bishop, K. Clements, C. Keitel, J. Kilpatrick y C. Laborde (Eds.), International handbook of mathematics education (pp. 205-237). Dordrecht, The Netherlands: Kluwer. https://doi.org/10.1007/978-94-009-1465-0_8

UNESCO (2020). ¿Qué se espera que aprendan los estudiantes de América Latina y el Caribe?: Análisis curricular del estudio regional comparativo y explicativo. Santiago: OREALC/UNESCO.

Vásquez, C. (2020). Educación estocástica: una herramienta para formar ciudadanos de sostenibilidad. Matemática, Educación y Sociedad, 3(2), 1-20.

Vásquez, C. \& García-Alonso, I. (en prensa). La educación estadística para el desarrollo sostenible en la formación del profesorado. Profesorado.

Vásquez, C. \& Rojas, F. (2020). Enseñar probabilidad para formar ciudadanos de sostenibilidad: ¿Qué sabemos de la COVID-19 y su propagación? UNO, 89, 22-29.

Wells, H. G. (1911). Mankind in the making. 5th ed. London: Chapman and Hall-[trabajo original de 1903]

Wild, C.J., Utts, J.M. \& Horton, N.J. (2018). What is statistics? En D. Ben-Zvi, K. Makar y J. Garfield (Eds.), International Handbook of Research in Statistics Education (pp. 5-36). Cham, Suiza: Springer. https://doi.org/10.1007/978-3-319-66195-7 1. 


\section{Autora}

\section{Claudia Vásquez}

Profesora de Matemática, Licenciada en Educación y Licenciada en Matemática por la Pontificia Universidad Católica de Chile; Magíster en Didáctica de la Matemática por la Pontificia Universidad Católica de Valparaíso; Magíster en Educación Matemática por la Universidad de La Frontera; Doctora en Ciencias de la Educación por la Universidad de Girona (España). Profesora Asociada de la Pontificia Universidad Católica de Chile. Investiga en la formación del profesorado, didáctica de la probabilidad, la estadística y la matemática y educación para el desarrollo sostenible. E-mail: cavasque@uc.cl. 